\title{
Option Pricing And Monte Carlo Simulations
}

George M. Jabbour, (Email: jabbour@gwu.edu), George Washington University

Yi-Kang Liu, (yikang@gwu.edu), George Washington University

\begin{abstract}
The advantage of Monte Carlo simulations is attributed to the flexibility of their implementation. In spite of their prevalence in finance, we address their efficiency and accuracy in option pricing from the perspective of variance reduction and price convergence. We demonstrate that increasing the number of paths in simulations will increase computational efficiency. Moreover, using a t-test, we examine the significance of price convergence, measured as the difference between sample means of option prices. Overall, our illustrative results show that the Monte Carlo simulation prices are not statistically different from the Black-Scholes type closed-form solution prices.
\end{abstract}

\section{INTRODUCTION}

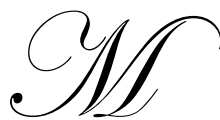

onte Carlo simulations (MCS) have recently been an important technique for option pricing in finance. MCS avoid complicated mathematics and have a straightforward implementation conceptually and practically. For example, to price a European down-and-out call barrier option ${ }^{1}$ by MCS, just treat it as a normal option unless the underlying asset price reaches the pre-determined level, as opposed to setting boundary conditions and solve a partial differential equation. In practice, MCS are procedures of sampling random outcomes for a particular process. However, while many academics and practitioners acknowledge the merits of MCS, some studies discuss their weaknesses in option pricing. Clewlow and Strickland (1998) and Hull (2000) point out that MCS generate high variances that lead to computational inefficiency. This problem can not be overlooked because such inefficiency may produce a biased estimator of the option price. In this paper, our focus is on the efficiency and accuracy of MCS in option pricing. We demonstrate that the estimated standard errors of MCS option prices can be reduced by increasing the number of paths in the simulations. Additionally, we use a t-test to examine whether MCS prices converge to Black-Scholes type of closed-form solution prices. The empirical evidence does not suggest any significant difference between those prices. Moreover, the results show that these two types of prices converge as the number of paths in simulations increases. The layout of this paper is as follows: section 2 provides a quick literature review. Section 3 examines variance reduction and price convergence of MCS. Section 4 provides the conclusions.

\section{LITERATURE REVIEW}

Originated from studies in physics, MCS have been very successfully applied in finance ${ }^{2}$. Hull and White (1987) use MCS to price options with stochastic volatilities. Schwartz and Torous (1989) apply MCS to the valuation of mortgaged-backed securities. Boyle et al. (1997) use MCS to price American options. On the other hand, the disadvantages of MCS are also discussed in some studies. Clewlow and Strickland (1998) and Hull (2000) argue that MCS are computationally inefficient due to the generated high variances.

\section{THE EFFICIENCY AND ACCURACY OF MONTE CARLO SIMULATIONS}

\section{Variance Reduction}

The efficiency of MCS increases with the number of paths used in the simulations. Since MCS are sampling random variables, option prices are random as well. The estimated standard error (ESE) is calculated as the sample

\footnotetext{
${ }^{1}$ A barrier option is a contingent claim whose payoff depends on whether the underlying asset has reached a certain pre-determined level for a specific path.

${ }^{2}$ See Jackel (2002) for a thorough summary of the applications of MCS in finance.
} 
standard deviation of MCS option prices $(S D)$ divided by the square-root of the number of paths $(m)$ :

$$
E S E=S D / \sqrt{m}
$$

From equation (1), ESE will decrease with an increase in the number of paths. Theoretically, if sample standard deviation of MCS option prices $(S D)$ is unchanged, when we increase $m$ from 100 to 400 , the $E S E$ should be reduced by $50 \%$. We demonstrate this negative relationship by using an example. The results are listed in Table 1 .

Table 1: Estimated Standard Errors of a European Put Option Price in Monte Carlo Simulations

The estimated standard error $(E S E)$ is given by: $E S E=$ sample standard deviation $(S D) / \sqrt{\text { number of paths }(m)}$

The input parameters are as follows: current stock price $s=10$, exercise price $\mathrm{x}=10$, time to maturity $\mathrm{t}=0.5 \mathrm{year}$, risk-free rate $\mathrm{r}=0.1$, stock return volatility $\sigma=0.4$, and $m=$ number of paths.

\begin{tabular}{|c|c|c|c|}
\hline & Case A $(m=100)$ & Case B $(m=400)$ & Case C $(m=2500)$ \\
\hline $\begin{array}{l}\text { Sample standard deviation of Monte Carlo simulation option } \\
\text { prices }(S D)\end{array}$ & 1.2874 & 1.2642 & 1.2407 \\
\hline $\begin{array}{l}\text { Estimated standard error of Monte Carlo simulation option prices } \\
(E S E)\end{array}$ & 0.1287 & 0.0632 & 0.0248 \\
\hline Theoretical standard error reduction* & 1 & 0.5 & 0.2 \\
\hline Practical estimated standard error reduction $* *$ & 1 & 0.4910 & 0.1926 \\
\hline
\end{tabular}

Table 1 shows the values of ESE for a hypothetical European put option for different paths. The theoretical standard error reduction in each case is defined as the square root of $m$ in base case divided by the square root of $m$ in the corresponding case. In addition, for comparison purposes, we calculate the practical estimated standard error reduction, defined as the $E S E$ of a case divided by the $S D$ of the base case. Table 1 clearly shows how the errors of option prices can be reduced as the number of paths increases. For example, ESE can be effectively reduced from $12.87 \%$ in case A (with 100 paths) to only $2.48 \%$ in case C (with 2500 paths). In addition, the theoretical standard error reduction ( $20 \%$ for case C) is very close to the practical estimated standard error reduction $(19.26 \%)$. The difference is due to the fact that the $S D$ is not the same in cases A and C.

\section{Price Convergence}

Price convergence is measured as the magnitude of the differences in sample means of two groups:

MCS and Black-Scholes type of closed-form solution prices. If these prices converge, the means of prices should be approximately the same. For illustration purposes, we choose path dependent options, specifically a down and out call option as our pricing target. We will limit our example to the European style to be consistent with the assumption of Black-Scholes type of closed-form solution.

In a Black-Scholes framework, the stock price follows a geometric Brownian motion. That is: 
$d S_{t}=r S_{t} d t+\sigma S_{t} d W_{t}$

where $S_{t}$ is the stock price at time $t, r$ is the risk free rate, $\sigma$ is the volatility, and $W_{t}$ is a Wiener process at time $t$

Using Wilmott (1998) approach, the value of a down and out European call option is given by:

$V(S, t)=C(S, t)-\left(\frac{S}{X}\right)^{-(k-1)} * C\left(\frac{X^{2}}{S}, t\right)$

where $k=2 r / \sigma^{2}, V(S, t)$ is the value of down and out European call option with underlying asset price $S$ and time $t, C(S, t)$ is the value of vanilla European call option with underlying asset price $S$ and time $t, X$ is the barrier price, and $C\left(X^{2} / S, t\right)$ is the value of vanilla European call option with underlying asset price $X^{2} / S$ and time $t$.

The European down and out call option is a path dependent option. In this sense, we need to check if the stock hits the threshold, a pre-set barrier price. If it does, the option ceases and has zero value. If not, the option survives and the final value of the underlying asset can be determined. Once we know the final value of the underlying asset, the payoff and the price of the barrier option can be calculated. By repeating the same procedure with various realizations, we can generate different samples which allow us to compare the relation between price convergence and the number of paths.

Figure 1 compares the MCS option prices with the Black-Scholes type of closed-form solution prices in three different cases with 100, 400, and 2500 paths. It also presents the intrinsic values - excess of stock price over exercise price or zero. Because down and out barrier option has zero value unless the underlying stock exceeds the barrier price, i.e. 8 in this example, we estimate the option value only when stock prices surpass the barrier. To examine the price convergence between MCS and Black-Scholes type prices, we apply a t-test to compare the means. We assume an exercise price of $\$ 10$, a time to maturity of six months, a barrier price of $\$ 8$, a risk-free rate of $10 \%$, and a volatility of $40 \%$. We use forty observations of MCS prices with respect to stock prices - by changing the underlying asset price from $\$ 8$ by $\$ .2$ until it reaches $\$ 16 .^{3}$

As shown in figure 1, it is easy to see that the MCS prices tend to converge to the Black-Scholes type of closed-form solution prices as the number of paths increases. To confirm this behavior, we test the following hypothesis:

H0: mean of MCS prices $=$ mean of closed-form solution (Black-Scholes type) prices

H1: mean of MCS prices $\neq$ mean of closed-form solution (Black-Scholes type) prices

The two-tail null hypothesis test is rejected if the t-statistic is larger than the corresponding critical value, 1.96, under a 95\% confidence interval. Table 2 summarizes the results. First we notice that the sample standard deviation of MCS option prices $(S D)$ decreases with the increase in the number of paths. This is consistent with the results in Table 1. Second, we find that all the t-statistics are less than the critical value 1.96. This result suggests that we can not find statistically significant evidence to reject the null hypothesis. Only at $10 \%$ level of significance (i.e. critical value 1.645) can the null hypothesis be rejected in the case of 100 paths. In practice, since the number of paths is usually larger than 100, it is reasonable to conclude that the MCS prices are not significantly different from the Black-Scholes type prices. Furthermore, we find the p-values increasing with the numbers of paths in simulations. The large p-value implies that the likelihood of failing to reject the false hypothesis (Type II error) is low. This indicates

\footnotetext{
${ }^{3}$ According to Griffiths et al. (1993), for applying a t-test, a sample size of thirty observations is considered large enough to satisfy the normal distribution assumption.
} 
Figure 1: The Relationship Between Monte Carlo Simulation Option Prices and Black-Scholes Type Prices

This figure depicts the intrinsic value, the Monte Carlo simulation option prices (denoted by MC) and the Black-Scholes type closed-form option prices (denoted by closed-form solution) of a European down and out call option with 100, 400, and 2500 paths. The input parameters are: barrier price $(x)=8$, time to maturity $(\mathrm{t})=0.5$, exercise price $(\mathrm{e})=10$, risk-free rate $(\mathrm{r})=0.1$, volatility $($ sigma $)=0.4$.

Diagram A (Number of paths $=\mathbf{1 0 0})$

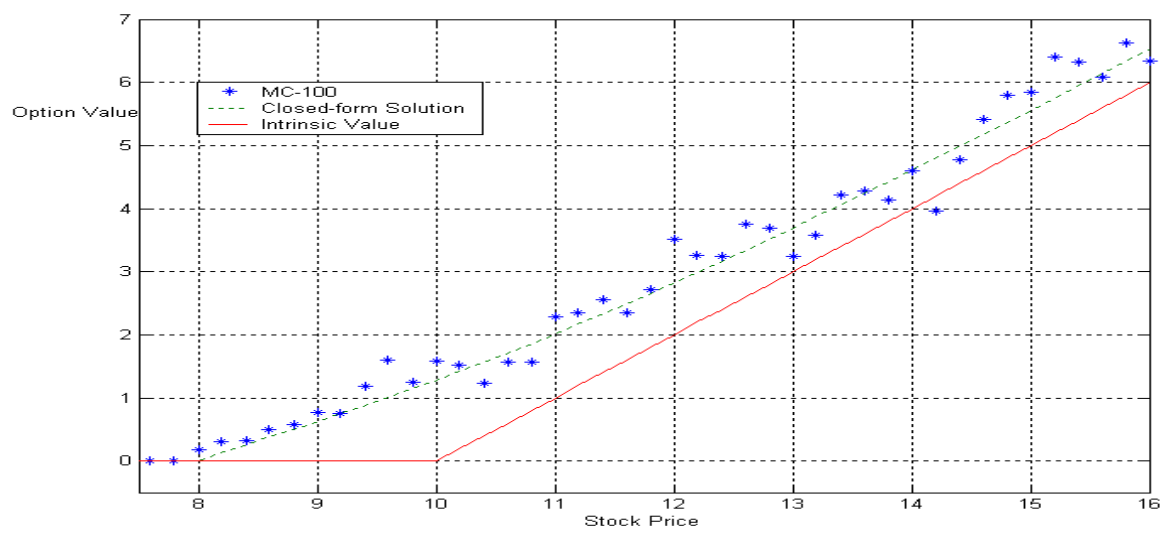

Diagram B (Number of paths $=400)$

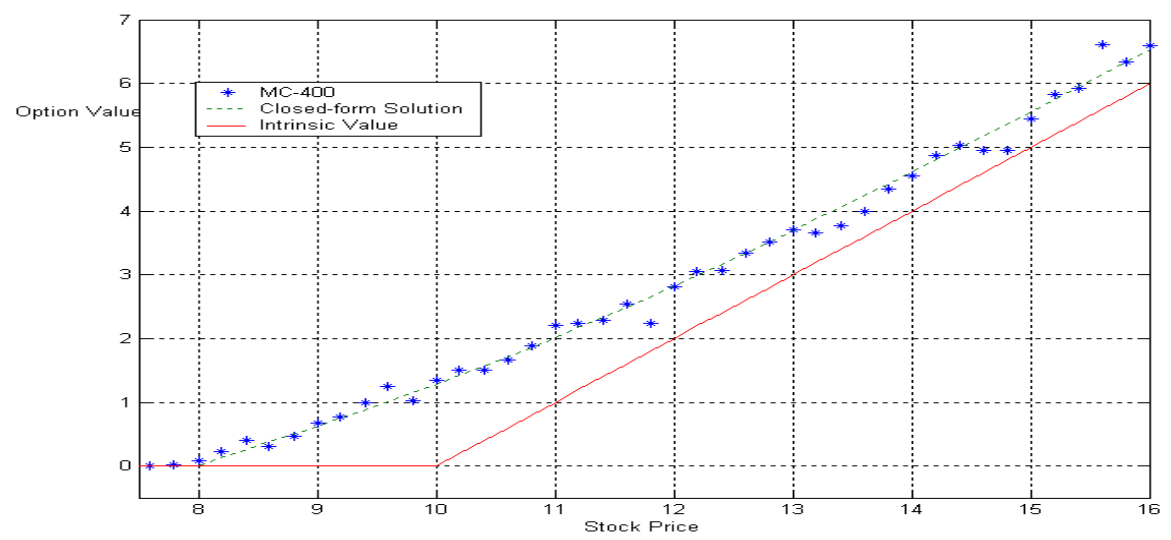

Diagram C (Number of paths $=2500$ )

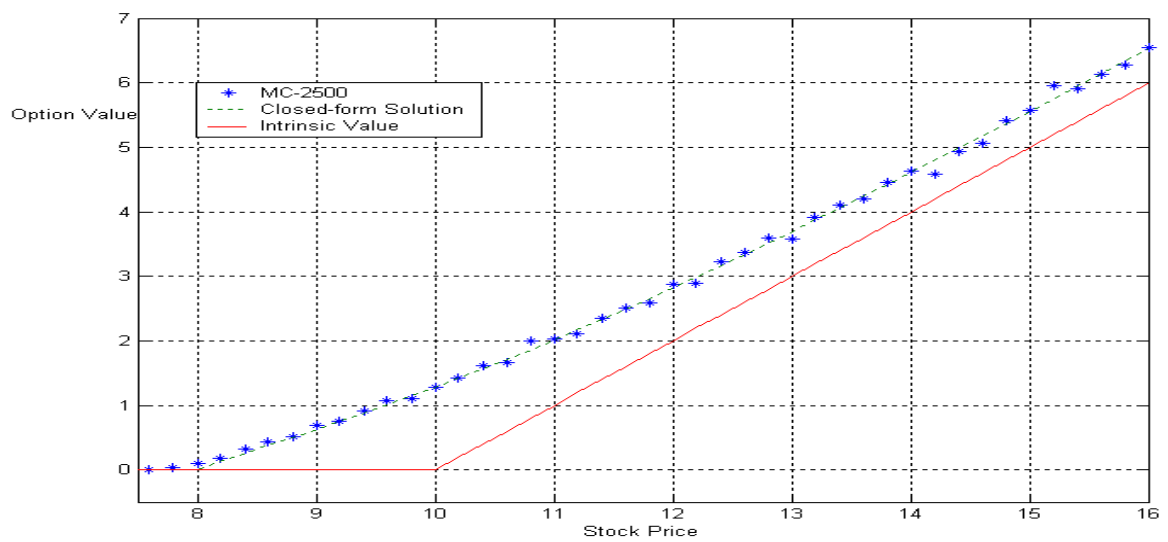


that the confidence level of our conclusion on not rejecting the null hypothesis increases with the p-value. In other words, the more simulations are executed, the more accurate the null hypothesis is, and the more evidence on the convergence of MCS prices to Black-Scholes type prices.

Table 2: Two-tail t Test for Price Convergence (sample size = 40)

This table shows any significant difference between Monte Carlo simulation prices and Black-Scholes type prices. The pricing target is a European down and out call option. The input parameters are: barrier price $(\mathrm{x})=8$, time to maturity $(\mathrm{t})=0.5$, exercise price $(e)=10$, risk-free rate $(r)=0.1$, volatility (sigma) $=0.4$. Case 1,2 and 3 are characterized by various numbers of paths in simulation, $m$. Each case has forty-paired observations. The null hypothesis for the two-tail test is:

H0: mean of MCS prices $=$ mean of closed-form solution (Black-Scholes type $)$ prices

H1: mean of MCS prices $\neq$ mean of closed-form solution (Black-Scholes type) prices

\begin{tabular}{|c|c|c|c|}
\hline & Case $1(m=100)$ & Case $2(m=400)$ & Case $3(m=2500)$ \\
\hline $\begin{array}{l}\text { Sample standard deviation of Monte Carlo simulation } \\
\text { option prices }(S D)\end{array}$ & 1.9478 & 1.9218 & 0.1947 \\
\hline Mean of the difference between MCS and BS type prices & 0.0870 & -0.0177 & 0.0057 \\
\hline Variance of the difference between MCS and BS type prices & 0.0942 & 0.0269 & 0.0054 \\
\hline Number of samples & 40 & 40 & 40 \\
\hline t-statistic/p-value* & $1.79 / 0.0806$ & $-0.68 / 0.5002$ & $0.49 / 0.6284$ \\
\hline Hypothesis testing** & $\begin{array}{l}\text { cannot reject } \\
\text { null hypothesis }\end{array}$ & $\begin{array}{l}\text { cannot reject } \\
\text { null hypothesis }\end{array}$ & $\begin{array}{l}\text { cannot reject } \\
\text { null hypothesis }\end{array}$ \\
\hline \multicolumn{4}{|l|}{ Under the null hypothesis, the t-statistic is estimated as follows: } \\
\hline
\end{tabular}

\section{CONCLUSIONS}

In this paper, we address the issues of efficiency and accuracy of Monte Carlo simulations in option pricing from the perspectives of variance reduction and price convergence. We demonstrate that increasing the number of paths in simulations will increase computational efficiency. Moreover, using t-test, we examine the tendency of price convergence, measured as the difference between sample means of option prices. Our results did not find significant evidence to reject the null hypothesis that the Monte Carlo simulation prices and the Black-Scholes type prices have the same mean.

\section{REFERENCES}

1. Clewlow, L. and C. Strickland, Implementing derivatives models, (1998), John Wiley \& Son Ltd.

2. Black, F. and M. Scholes, (1973), The pricing of options and corporate liabilities, Journal of Political Economy, 81, 637-654.

3. Boyle, P., M. Broadie, and P. Glasserman, (1997), Monte Carlo methods for security pricing, Journal of Economic Dynamics and Control, 21, 1267-1321.

4. $\quad$ Griffiths, W., R. C. Hill, and G. Judge, Learning and practicing econometrics, (1993), John Wiley \& Son Ltd.

5. Hull, J., Options, futures and other derivatives, fourth edition,(2000), Prentice-Hall Inc.

6. Hull, J. and A. White, (1987), The pricing of options on assets with stochastic volatilities, Journal of Finance 42, 281-300.

7. Jackel, P., Monte Carlo method in finance, (2002), John Wiley \& Son Ltd. 
8. Schwartz, E. and W. Torous, (1989), Prepayment and the valuation of mortgaged-backed securities, Journal of Finance 44, 375-392.

9. Wilmott, P., Derivatives: the theory and practice of financial engineering, (1998), John Wiley \& Son Ltd.

\section{NOTES}

